\title{
Conceptual design of the W7-X port liners
}

\author{
Reinhold J. Stadler ${ }^{\mathrm{a}}$, A. Peacock ${ }^{\mathrm{a}}$, J. Boscary ${ }^{\mathrm{a}}$, B Mendelevitch ${ }^{\mathrm{a}}$, P. Scholz $^{\mathrm{b}}$, W. Schubert ${ }^{\mathrm{a}}$ \\ ${ }^{a}$ Max Planck Institute for Plasma Physics, 85748 Garching, Germany \\ ${ }^{b}$ Max Planck Institute for Plasma Physics, 17491 Greifswald, Germany
}

\begin{abstract}
The experimental stellarator Wendelstein 7-X has a large variety of ports for plasma diagnostics. For the steady-state operation with $10 \mathrm{MW}$ input power and a plasma pulse length of up to 30 minutes, 101 diagnostics ports need to be shielded with actively cooled port liners made of stainless steel. A total of 41 variants of port liners taking into account the various port geometries and the interfaces with different types of diagnostics have to be installed. Before starting the production, different concepts have been studied. Five full-scale demonstrators and three different technologies representative of the port liner variety have been designed and are being produced. Results of this fabrication will serve as a basis for the procurement of the 101 port liners.
\end{abstract}

Keywords: Stellarator, Wendelstein 7-X, In-Vessel Components, First Wall, Port Liner.

\section{Introduction}

After two preliminary stages of operation at lower input power and shorter pulse length, the stellarator Wendelstein 7-X (W7-X) will be finally operated to its nominal parameters of $10 \mathrm{MW}$ input power for plasma pulse lengths of up to 30 minutes and peak power with additional combined heating systems of up to $24 \mathrm{MW}$ for $10 \mathrm{~s}$ [1]. W7-X has 244 ports for heating, water-cooling [2] and plasma diagnostics. Among them, 101 diagnostics ports needs to be shielded with actively cooled port liners, which will only be installed or possibly adapted for the full long pulse operation.

Before starting the production of the port liners, a conceptual design phase has been carried out. Different concepts have been developed based on the experience gained during the successful fabrication of the panels of the first wall [3], which have already been installed in the plasma vessel of W7-X. The main difficulties with the design of these port liners are the variety of ports with different cross-sections, sizes and lengths, and the interface with very different types of diagnostics. To minimize the risks of the production and to reduce the associated costs, much effort has been invested in a preliminary development phase which aimed at reducing the number of port liner variants. This was particularly difficult since some diagnostics are still in a very early conceptual design stage. Finally, a total of 41 port liner variants have been defined. Using these as a basis, five full-scale demonstrators have been fully designed and these will be each produced using one of three selected technologies, best suited to the geometry of the particular port liner. This process will be discussed here.

\section{Description and function of the port liner}

A large variety of ports is available in $\mathrm{W} 7-\mathrm{X}$ for the study of the plasma from different locations along the helically-shaped plasma with a 5-fold symmetry and a varying cross-section. An example of a port is shown in Fig. 1. The wall thickness of the stainless steel port is 3 to
$15 \mathrm{~mm}$. The length of the ports varies from 1.52 to $2.28 \mathrm{~m}$. Most of the ports have a circular cross section with an internal diameter varying from 136 to $386 \mathrm{~mm}, 8$ ports are rectangular and 13 are oval. The ports are welded at one extremity to the plasma vessel and at the other connected via bellows to the cryostat. The bellows accommodate relative movement between the plasma vessel and the cryostat. Flanges, generally used for diagnostics, are placed on the cryostat side. Due to limited space availability, the walls of the diagnostic ports are not cooled. The ports go through the cryogenic vacuum of the cryostat which contains the superconducting magnetic coils. A view from the plasma vessel to a typical port (AEF) is shown in Fig. 2. To minimize the heat transfer to the cryostat, a multi-layer insulation covers the external sides of the ports. A maximum port wall temperature of $75^{\circ} \mathrm{C}$ leads to a thermal loss of $90 \mathrm{~W} / \mathrm{m}^{2}$ through the multi layer insulation (MLI) to a $70 \mathrm{~K}$ cold thermal shield. These limits should not be exceeded. To fulfill this limit, an actively cooled thermal protection of the port wall, called a port liner, is required for full long pulse operation.

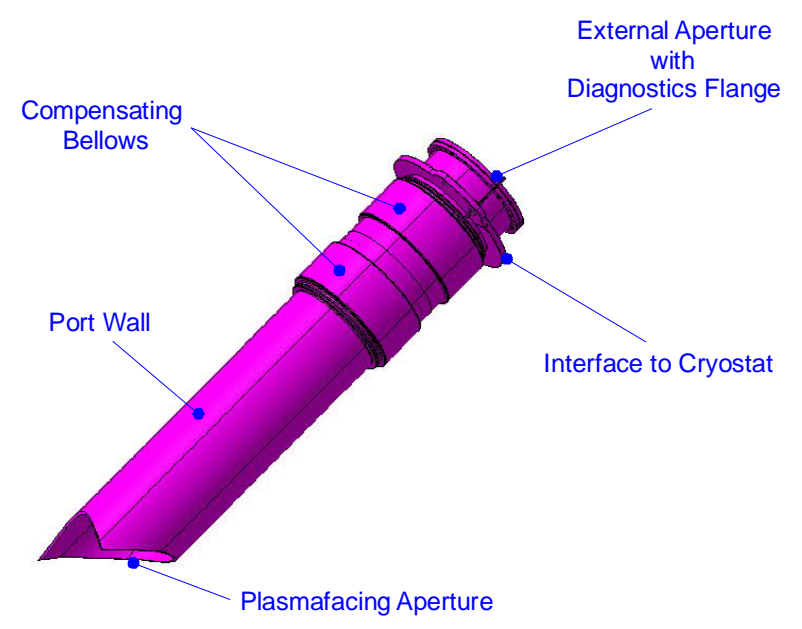

Fig. 1. Example of diagnostic port layout (port AEF). 


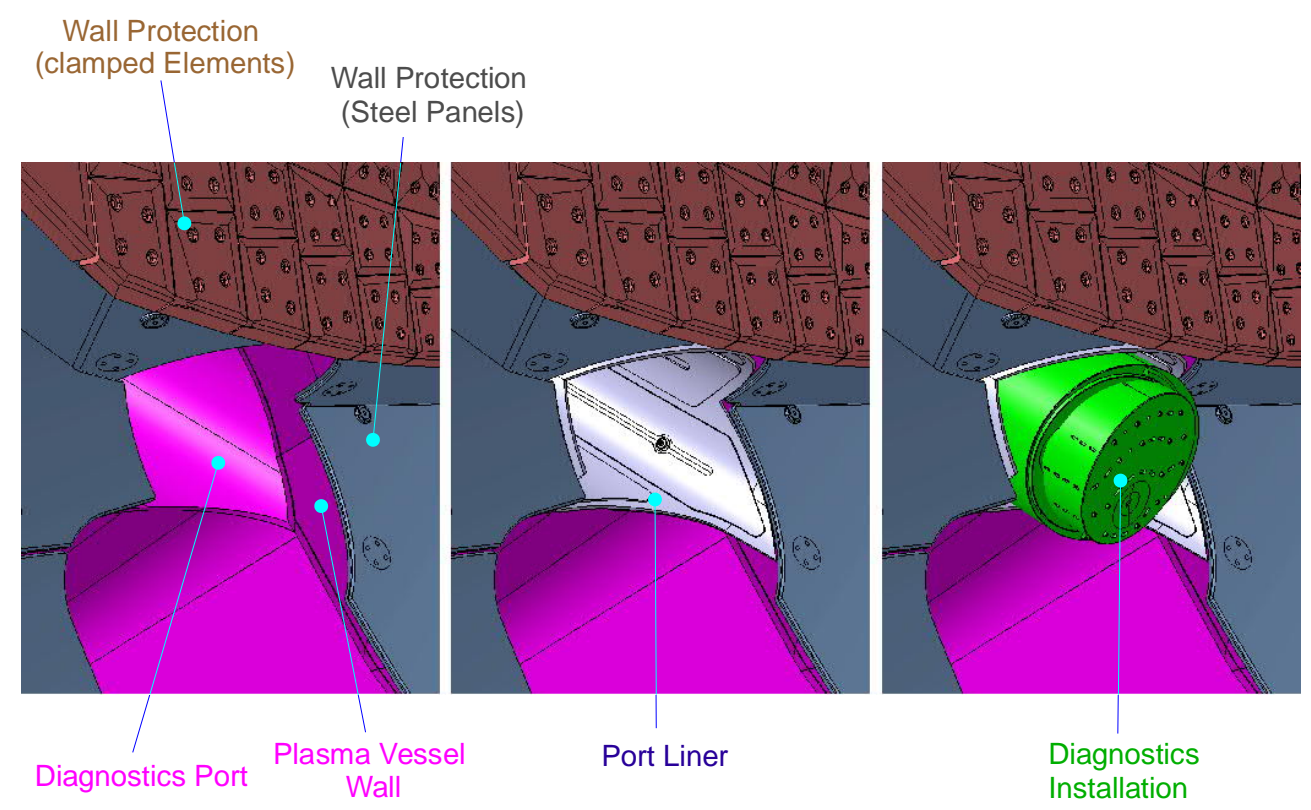

Fig. 2. Example of port installation situation (port AEF). On the left are shown the plasma vessel and port, together with wall protection components. The center picture shows the installed port liner, ending flush with the wall protection. At right is shown the complete situation, with the installed diagnostics.

The port liner is a cylindrical shell, with incorporated cooling channels. At the plasma-facing aperture the port liner shape matches closely the opening in the wall protection components, so that only a small gap remains. At the flange side the cooling supply lines are mounted, which connect the port liner to the external periphery (see Fig. 6). The length of the port liner into the port depends on the level of distributed thermal load along the port length. Attachment points are integrated in the shell, to mount and position the port liner within the port.

The port liner protects the port wall against plasma radiation loads and stray radiation from the Electron Cyclotron Resonance Heating (ECRH) system. The plasma radiation load at the plasma facing aperture of the port is estimated to an average load of $100 \mathrm{~kW} / \mathrm{m}^{2}$, maximum local values can reach $200 \mathrm{~kW} / \mathrm{m}^{2}$. Based on this, the design load for the port liner surfaces, with its orientation perpendicular to the plasma, was derived. The port liners are designed for a maximum load of $100 \mathrm{~kW} / \mathrm{m}^{2}$ at the port opening, which is decreasing along the port as the distance to the plasma increases. An additional thermal load comes from the absorption of ECRH stray radiation; using an absorption coefficient of $2 \%$ for stainless steel results in a maximum estimated absorption of $1 \mathrm{~kW} / \mathrm{m}^{2}$.

Since the port liners are integrated in the diagnostic ports they must not restrict the diagnostic operation by limiting lines of sight or colliding with the diagnostics. A function of some of the port liner is also to thermally protect sensitive diagnostic parts. In a few cases the diagnostic components may be integrated into the port liner.

\section{Technology options of the port liner}

Three technologies have been selected as promising candidates for the various port liner geometries (Fig. 3):

- Technology 1: The cooling channels are machined into a $5 \mathrm{~mm}$ thick base plate, which is closed by a welded $2 \mathrm{~mm}$ thick steel cover. This solution is suitable to rectangular ports.

- Technology 2: Two 2mm thick steel plates are welded together and inflated by overpressure to form the cooling channels. This technology has been tested successfully on a preliminary prototype.

- Technology 3: A $1.5 \mathrm{~mm}$ thick cover sheet is welded to a $5 \mathrm{~mm}$ thick base sheet and the cooling channel formed by inflating with internal overpressure. This is the same technology as was used for the first wall panels [3].

The resulting geometry of the cooling channel as a function of the technology is shown in Fig. 3: a square channel for technology 1 , an oval channel for technology 2 and a half-oval channel for technology 3. These technologies have been successfully applied to some of the very special port liners. A total of 5 port liners will be installed in the machine for the start of operation. Fig. 4 shows the port liner of a twin port diagnostic used for the Thomson scattering diagnostic. In this case, the port liner 
is also a carrier structure for the diagnostic shutter. This diagnostic port liner was developed and manufactured by an industrial team of the MDT company, Deggendorf, Germany.

Material to be used for the port liner must comply with W7-X standards. The stainless steel is DIN 1.4435, with a low Co-content $(<500 \mathrm{ppm})$ and a low magnetic permeability $<1.03$ and $<1.05$ for the material and the seam weld, respectively.

\section{Conceptual design of the port liner}

The port liners are designed to be actively watercooled. The coolant will be used for heat removal during plasma operation (inlet temperature of $15-30^{\circ} \mathrm{C}$ ) and for the baking of the component (inlet temperature of $150^{\circ} \mathrm{C}$ ).
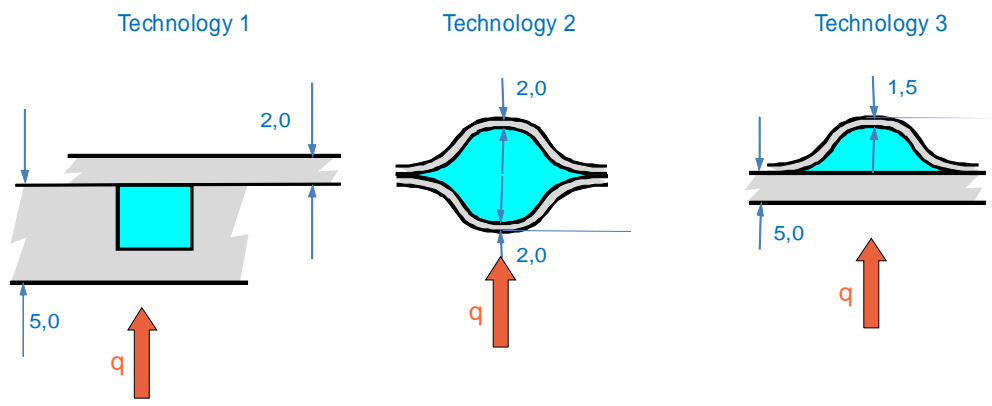

Fig. 3. Technologies used for the port liner.

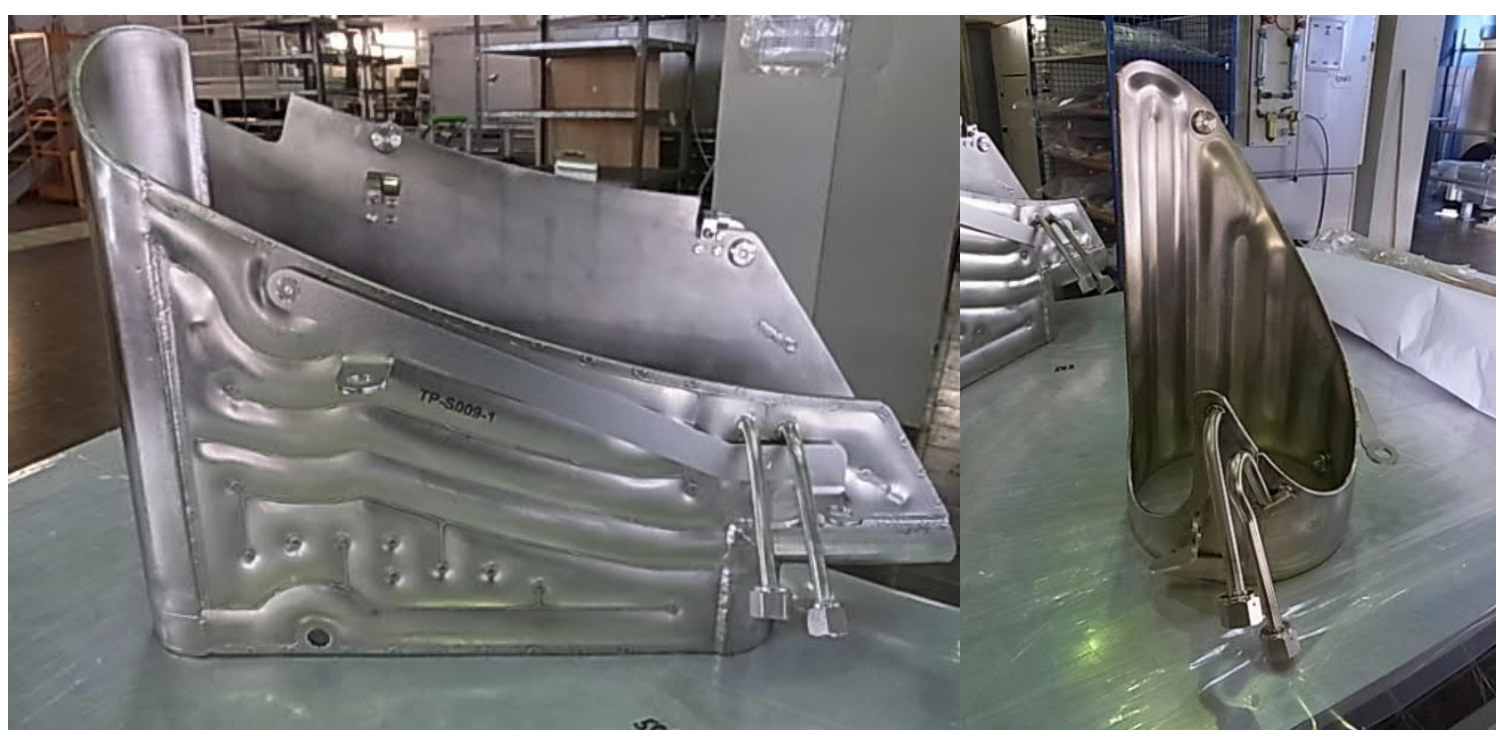

Fig. 4. Twin diagnostic port liner (twin port AEM31 / AEN31).

The total pressure drop specified for the components (diagnostic + port liner + supply lines + connections) inside the port is $1 \mathrm{MPa}$ at the port flange with an average flow rate of $0.21 / \mathrm{s}$. Inlet pressure is $2.5 \mathrm{MPa}$. For the port liner and its supply lines, a maximum pressure drop of $0.6 \mathrm{MPa}$ has been specified. The temperature increase during full pulse operation shall not exceed $50 \mathrm{~K}$, with a maximum water outlet temperature of $80^{\circ} \mathrm{C}$.

The port liners will operate in ultra-high vacuum and as for all in vessel components [4], the produced components have to pass a pressurized Helium leak test with leak rates $<5.10^{-7} \mathrm{~Pa} 1 / \mathrm{s}$ at $20^{\circ} \mathrm{C}$ and $3.5 \mathrm{MPa}$, and $<5.10^{-6} \mathrm{~Pa} 1 / \mathrm{s}$ at $150^{\circ} \mathrm{C}$ and $2.5 \mathrm{MPa}$. The outgassing rate should be $<1 * 10^{-5} \mathrm{Pal} / \mathrm{s} \mathrm{m}^{2}$. The technology must ensure a burst pressure in excess of $7 \mathrm{MPa}$ at $20^{\circ} \mathrm{C}$, which will be experimentally demonstrated with dedicated full-scale elements.

The cooling water, supplied from the outside, is fed by a set of pipes and flexible hoses, which allow for installation tolerances and relative deflection resulting from operational loads and in particular the large relative movement of the diagnostic bellows. The supply lines are connected to the diagnostics flange or a separate extra ring, depending on the geometrical arrangement of the diagnostic system.

The position and dimensions of the port liner are defined by the diagnostics, port wall and first wall 
protection components at the plasma facing aperture. The available space for the installation of the port liners is restricted to $15 \mathrm{~mm}$, as they have to fit in the small gap between diagnostics and port wall. The attachment of the port liner to the port wall is achieved by a set of connectors, installed in the port liner. There is a fixed point close to the plasma vessel, and sliding points at the other end of the port liner (Fig 5).

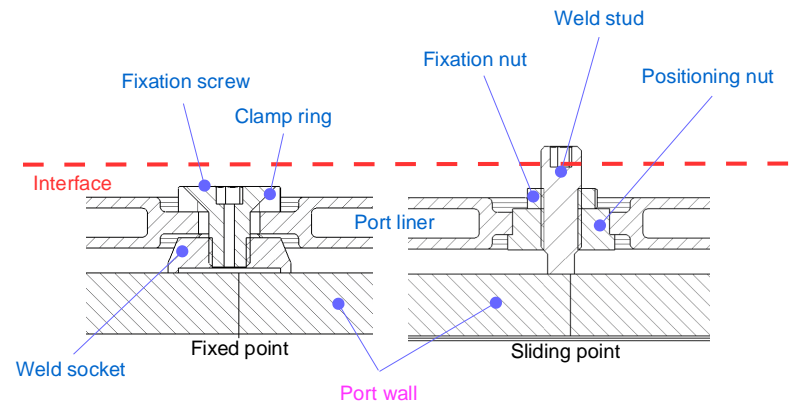

Fig. 5. Conceptual layout of port liner attachment.

These allow adaptation of the port-liner position to best fit the as built plasma vessel and wall protection geometries. The fixtures also allow for relative expansion between port and port liner during operation.

An example of the conceptual design of the port liner to be installed inside the AEF port with a diagnostic immersion tube is shown in Fig. 6. In this case, the cooling is supplied with a separate ring. This design also illustrates the complexity of the design at the plasma vessel interface, which requires an irregular end shape, the geometrical intersection between the port and the plasma vessel being oval.

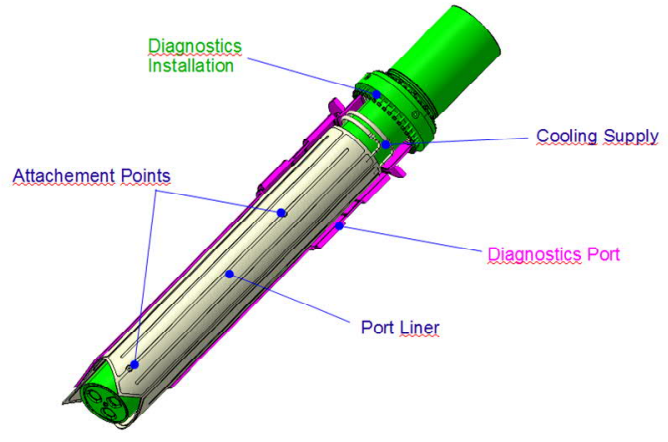

Fig. 6. Installation of port liner (port AEF).

An extensive review of the 101 diagnostic ports which require a port liner has been conducted. After much iteration, a total of 41 variants have been defined. For each port and associated diagnostic a concept of the port liner layout has to be established to check the interfaces. The technology selected for each port liner type depends mainly on the geometrical constraints.

\section{Implementation}

\subsection{Work package 1: Full scale demonstrator}

The large number of shapes and sizes excludes the possibility of manufacturing a prototype for each variant. Therefore, a set of five full scale demonstrators has been selected, which are representative of all the variants (Table 1). These prototypes will be used to validate the technologies and the acceptance criteria for the production. The demonstrators are cooled over the whole port length, as this is the most demanding configuration. However, first preliminary calculations show a potential that the rear part of the port liner could be uncooled due to the reduced thermal loading in this area.

Table 1: description of demonstrators

\begin{tabular}{|c|c|c|}
\hline Demonstrator Type & Geometry & Technology \\
\hline AEEv3 & Oval 970x370mm & 2 \\
\hline AEF & Circular Ø264mm & 2 \\
\hline AEIv2 & Circular Ø170mm & 1 \\
\hline AEOv2 & Oval 570x120mm & 1 and 3 \\
\hline AEZ & Circular Ø94mm & 3 \\
\hline
\end{tabular}

This could significantly reduce the complexity of the future fabrication of the port liners. The five demonstrators will have to pass different inspections: Geometrical stability as well as thermal performance will be a key for successful operation of the port liners. The established manufacturing procedures will be then applied and adapted, if required, to the other variants. This knowledge will be used to identify possible standardization of the components in order to reduce development and manufacturing efforts. This stage will validate the conceptual design phase before the release of the fabrication. At present the bidding process for the procurement of the demonstrators is running.

\subsection{Work package 2: production of 101 port liners}

The expertise gained during the demonstrator phase will be applied to the detailed design of the serial port liners. The present schedule assumes a set of three to five manufacturing lots, which will be delivered in sequence. This allows a balanced workload for incoming inspection, testing and acceptance of the components.

\section{Conclusion}

101 port liners with 41 variants have to be installed in the diagnostic ports for the full long pulse operation of W7-X. The procurement of the port liners is divided into two successive work packages. In work package 1, the selected technologies will be validated with the fabrication and extensive testing of five full-scale demonstrators. Their design has been completed and the procurement started. The conceptual design phase of the remaining port liners is not yet completed and will be finalized as a result of the demonstrator testing. Work package 2 is the production of the 101 port liners, which are planned to be delivered to W7-X in 2018 for installation. 


\section{Acknowledgments}

This project has received funding from the European Union's Horizon 2020 research and innovation programme under grant agreement number 633053 . The views and opinions expressed herein do not necessarily reflect those of the European Commission.

\section{References}

[1] M. Gasparotto, C. Baylard, H. S. Bosch, D. Hartmann, T. Klinger, R. Vilbrandt et al., Wendelstein 7-X - Status of the project and commissioning planning, Fus. Eng. and Des. 89 (2014) 2121-2127.

[2] B. Mendelevitch, A. Vorköper, J. Boscary, A. Cardella, F. Hurd, Ch. Li et al., Design analysis and manufacturing of the cooling lines of the in vessel components of W7-X, Fus. Eng. and Des. 86 (2011) 1669-1672.

[3] A. Peacock, A. Girlinger, A. Vorköper, J. Boscary, H. Greuner, F. Hurd et al., The procurement and testing of the stainless steel in-vessel panels of the Wendelstein 7-X Stellarator, Fus. Eng. and Des. 86 (2011) 1706-1709.

[4] R. Stadler, et al., "The in-vessel components of the experiment Wendelstein 7-X”, Fus. Eng. and Des. 84 (2-6) (2009) 305-308. 